\title{
Demonstration of Kerr Nonlinearity in Silicon Microcylindrical Resonators
}

\author{
Natasha Vukovic*, Noel Healy, Priyanth Mehta, and Anna C. Peacock \\ Optoelectronics Research Centre, University of Southampton, Southampton SO17 IBJ, UK \\ *ntv@orc.soton.ac.uk
}

\begin{abstract}
We investigate the Kerr nonlinearity in a-Si:H based microcylindrical resonators. The large resonant wavelength shift observed for pulsed excitation is used to demonstrate ultrafast alloptical switching.

OCIS codes: (140.4780) Optical Resonators; (140.3948) Microcavity Devices; (190.3270) Kerr Effect; (160.6000) Semiconductor Materials.
\end{abstract}

\section{Introduction}

Whispering gallery mode (WGM) microresonators with ultra-high quality factors $(Q)$ and small mode volumes have been demonstrated in a range of geometries and materials. Their unique method of mode confinement makes them ideal for the examination and exploitation of optical functionalities such as lasing, cavity quantum electrodynamics and nonlinear optics. In more recent experiments, silicon microresonators based on planar microelectronics technology have been used to demonstrate all-optical switching through nonlinear processes. These devices have typically used the thermo-optic nonlinearity or free-carrier absorption of silicon to produce a modulated signal [1]. However, modulation speeds are severely limited due to the slow thermal response time and long free carrier lifetime of silicon. In order to achieve ultrafast all-optical switching through a nonlinear process it is desirable to use the near instantaneous Kerr effect [2,3].

In this paper we investigate the Kerr nonlinearity in a hydrogenated amorphous silicon (a-Si:H) microresonator that has been fabricated from the silicon optical fiber platform. We use a simple pump-probe experiment to observe and compare the resonant wavelength shift caused when the microresonator is pumped with a continuous wave (CW) and a picosecond pulsed source. For the same average power, the high peak power pulse source induces a much larger wavelength shift so that the Kerr and thermal nonlinearity contributions can be deconvolved. We use the results to show that for picosecond pulses acting as the pump, the modulation bandwidth of the resonator is limited by the cavity photon lifetime.

\section{Fabrication and characterization}

The silicon microresonator was formed by etching the silica cladding away from an a-Si:H core fiber fabricated using a high pressure deposition technique, as described in Ref. [4]. The fiber had a $5.6 \mu \mathrm{m}$ diameter core and a linear loss of $2.5 \mathrm{~dB} / \mathrm{cm}$ at $1.55 \mu \mathrm{m}$, measured via the cutback method. A tapered single mode fiber was used to probe the resonator's optical properties over an extended telecoms band; the experimental setup which uses just a single scanning CW laser is represented by the white boxes in Fig. 1(a). The transmission spectra presented in Fig. 1(b) and Fig. 1(c) show the sharp resonances associated with the resonator.
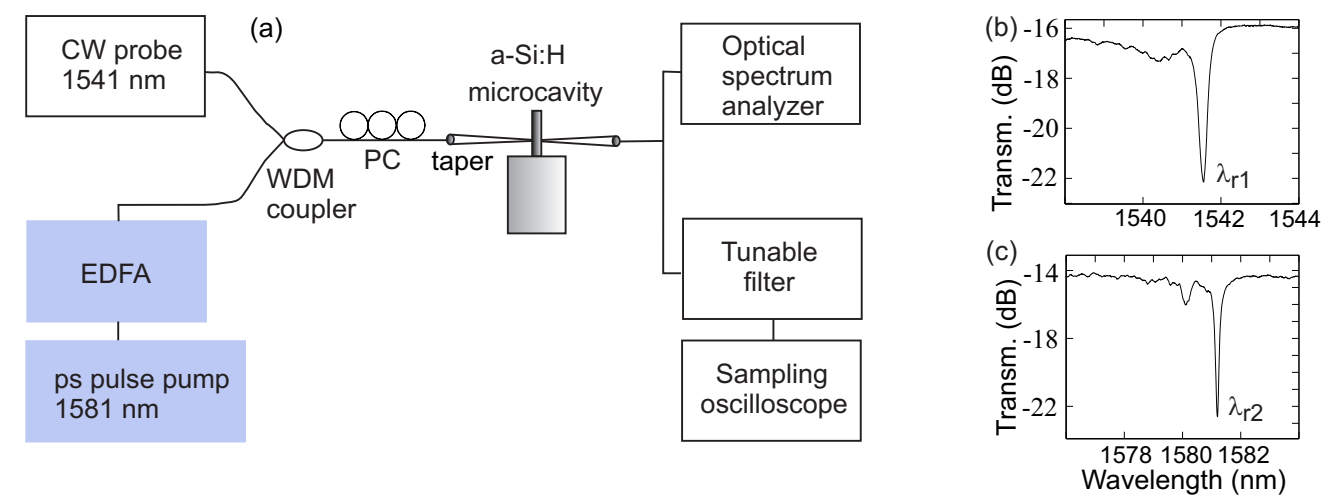

Fig. 1. (a) Experimental setup used to characterize Kerr effect in a-Si:H microcylindrical resonator. Transmission spectrum showing two resonances (b) $\lambda_{\mathrm{r} 1} \sim 1541.5 \mathrm{~nm}$, with measured loaded $Q$ factor $Q_{r 1}=6.1 \times 10^{3}$, and (c) $\lambda_{\mathrm{r} 2} \sim 1581.2 \mathrm{~nm}$, with $Q_{r 2}=7.9 \times 10^{3}$. 


\section{Results and discussion}

Using the scanning tunable $\mathrm{CW}$ laser, we measured the resonant shift as a function of power coupled into the resonator $\left(\mathrm{P}_{\mathrm{c}}\right)$, and the results are shown in Fig. 2(a). The linear absorption of the resonator manifests itself as a red shift of the resonant wavelength and this shift is linearly proportional to the coupled power up to $\sim 70 \mu \mathrm{W}$. Above this threshold the nonlinear effects in the resonator start to contribute to the red shift, which results in a deviation from the linearity of the power/wavelength shift. To investigate this further, the setup is modified to include the pulsed source (blue boxes) in Fig. 1(a). The picosecond tunable pulse pump (100ps/40 MHz) was co-propagated with the scanning laser and the resonant wavelength shift was measured for a range of coupled powers up to $20 \mu \mathrm{W}$, the results of which are shown in Fig. 2(b). The figure shows a $\sim 2 \mathrm{~nm}$ resonant wavelength shift at the max coupled power of $20 \mu \mathrm{W}$. We then replaced the pulsed source with the tunable CW source set at the pump wavelength and measured the shift for the same range of average powers. A resonant shift of only $\sim 0.2 \mathrm{~nm}$ was observed when the power coupled into the resonator is $\sim 20 \mu \mathrm{W}$, which can be attributed to the thermal nonlinearity. This order of magnitude difference in the shifts is a result of the Kerr nonlinearity induced by the high peak powers of the pulse source. As the pump pulses are much shorter than the thermal response time of the microresonator, $\tau_{\theta} \sim 7.5 \mu \mathrm{s}$ [4], it can be concluded that the instantaneous Kerr effect is the prevailing nonlinear mechanism.
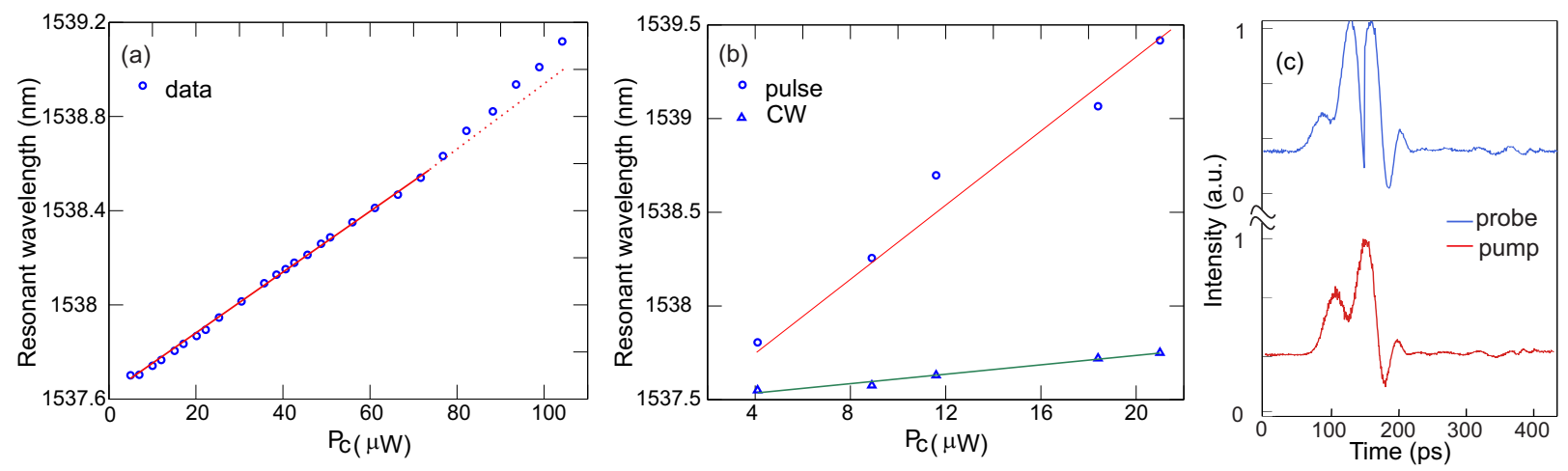

Fig. 2. (a) Resonant wavelength as a function of coupled power at CW scanning. (b) Resonant wavelength as a function of coupled power for pulsed (circle) and CW (triangles) pump regimes. (c) Temporal response measured by the sampling scope.

To exploit the Kerr effect for all optical switching, the two tunable lasers, CW and pulsed, were tuned to the resonances $\lambda_{\mathrm{r} 1}$ (probe) and $\lambda_{\mathrm{r} 2}$ (pump), respectively, and launched into the tapered fiber. The optical average power of the pump was $9 \mu \mathrm{W}$, which corresponds to a pulse energy of $0.22 \mathrm{pJ}$. A tunable filter was used at the output to isolate the probe, which was monitored using a $30 \mathrm{GHz}$ sampling oscilloscope. Fig. 2(c) illustrates the observed ultrafast all-optical switching that occurs when the Kerr induced index change shifts the resonator on and off resonance with the probe. Although the modulation extinction ratio is only $1 \mathrm{~dB}$, this proof of concept experiment is subject to ongoing optimization. In principle, the Kerr effect allows one to realize almost arbitrary modulation speed due to its instantaneous response. However, in practice the speed is limited by the $-3 \mathrm{~dB}$ bandwidth of the resonator, governed by $B=c /\left(\lambda_{r} Q_{l}\right)$, where $c$ is the speed of light in vacuum and $Q_{l}$ is the loaded quality factor. For the measured $Q_{l}=7.9 \times 10^{3}$, the switching speed is determined as $\sim 24 \mathrm{GHz}$. This switching speed can be further increased by reducing the $Q_{l}$, by adjusting the concentration of the incorporated hydrogen in the resonator.

In conclusion, the first demonstration of the Kerr effect in a-Si:H fiber based microresonators is presented. We show that the power threshold needed to induce Kerr nonlinearity is only a few $\mu \mathrm{W}$ in a picosecond pulse excitation regime. The near instantaneous Kerr effect shown in an a-Si:H microresonator opens the route towards devices with all-optical functionalities such as filtering, switching and modulation.

The authors thank the Department of Chemistry and Materials Research Institute of the Pennsylvania State University and Todd D. Day for the fabrication of the silicon fiber.

\section{References}

[1] M. Lipson, “Switching light on a silicon chip,” Opt. Mater. 27, 731-739 (2005).

[2] H. Rokhsari and K. J. Vahala, “Observation of Kerr nonlinearity in microcavities at room temperature,” Opt. Lett. 30, 427-429 (2005).

[3] M. Pöllinger and A. Rauschenbeutel, “All-optical signal processing at ultra-low powers in bottle microresonators using the Kerr effect,” Opt. Express 18, 17764-17775 (2010).

[4] N. Vukovic, N. Healy, T. D. Day, P. Horak, P. J. A. Sazio, J. V. Badding, and A. C. Peacock, "Ultra smooth microcylindrical resonators fabricated from the silicon optical fiber platform," Appl. Phys. Lett. 99, 031117-3 (2011). 